\title{
Examples of acquisition and application of biooptical signals
}

\author{
A. Cysewska-Sobusiak* \\ Division of Metrology and Optoelectronics, Institute of Electrical Engineering and Electronics, \\ Poznan University of Technology, Piotrowo 3a, 60-965 Poznan
}

Received May 30, 2019; accepted June 15, 2019; published June 30, 2019

\begin{abstract}
Scientific activity of Division of Metrology and Optoelectronics at Poznan University of Poland (PUT) includes methods and systems used in modern electronic, optoelectronic, and biomedical metrology. Noninvasive diagnostic methods applied in medical engineering, with a special interest in optoelectronic sensor technology and advanced methods of imaging, are considered in the paper. The author, who was the Head of the Division, presents a short review of some selected former results of studies related to biophotonics.
\end{abstract}

In the years 1999-2017 the author of the paper led the Division of Metrology and Optoelectronics with PUT. She built with success a scientific team which major research area focuses on the following directions:

- Metrological problems in interdisciplinary investigations with a special interest in noninvasive biomeasurements and advanced imaging methods.

- Acquisition, processing and measurements of optical and biooptical signals.

In this period seven young members of the Division team obtained their $\mathrm{PhD}$ degrees in electronic, optoelectronic, and biomedical metrology. Scientific research is combined with teaching. Interests include research and development of measuring systems, evaluation of uncertainty of measurement results, and improvement of instrumentation used in bio-measurements [1, 6-14]. Our investigations in biophotonics are mainly based on utilization of light-tissue interaction (Fig. 1).
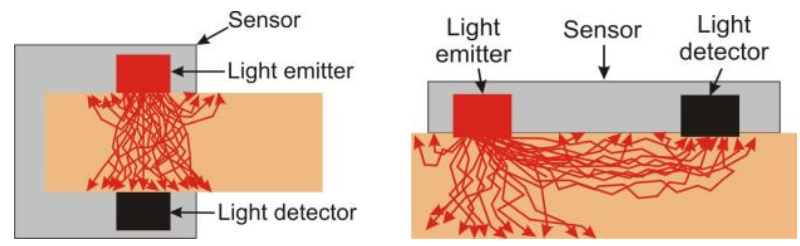

Fig. 1. Two variants of light-tissue interaction to be used in two types of sensors: transmission variant (left), and reflectance variant (right).

The noninvasive optoelectronic sensor consisting of an input light emitter and an output light detector can give an electrical signal that is proportional to light intensity which is transmitted or reflected by examined body sites. The sensor should be equipped with a highly efficient

\footnotetext{
*E-mail: anna.cysewska@put.poznan.pl
}

incident light emitter (containing either a single LED or a set of LEDs) and a sensitive output light detector (containing either a single photodiode or a set of photodiodes). The modulations of biooptical signals are induced in tissues by arterial blood pulsations. These modulations are the basis of very important optical bloodless diagnostic methods: photoplethysmography (PPG) and pulse oximetry [1-4] that make it possible to monitor living tissues vitality noninvasively.

Depending on the locality and diameter of the light beam passing through an object, the effects of light-tissue interaction can differ considerably. Optical radiation that is to play the role of an effective information carrier should be sufficiently coherent and, due to the high optical density of the object, should also have a possibly high intensity. However, for higher power density quantities, some destructive photothermal effects may occur. The wavelength and power of radiation selected improperly may not only act ineffectively but may cause damage or destruction of the object.

Photonic techniques allow us to use the optical properties of transilluminated or illuminated tissues or organs for their imaging. These properties depend on strong light absorption and scattering [5]. Tissues are optically turbid media that are highly scattering. A typical scattering coefficient for visible light in human tissues is $1001 / \mathrm{cm}$ in comparison with $0.21 / \mathrm{cm}$ for $\mathrm{X}$ rays used in medical diagnostics. Transillumination windows extends from 600 to $1200 \mathrm{~nm}$. The major difference caused by oxygen lies in the red band from 600 to $750 \mathrm{~nm}$. Numerous issues related to result interpretation remain unsolved. There are specific inverse problems in modeling and simulation of light transmission through or reflection by tissue sets. The answer of such an object is known, but the cause can be unknown. The inverse problems are complex because more than one tissue composition may share the same resultant intensity transmittance or reflectance [6].

Biooptical signals can be acquired with optoelectronic sensors placed on peripheral body sites. From the measurement point of view, especially convenient for effective transillumination are fingers, which are widely used as objects in the transmission variants of the 
mentioned pulse oximetry and photoplethysmography. Of course, like other human body parts, the structure of fingers is also complex and there is heterogeneity in volume. The examples of finger cross-sections shown in Fig. 2 illustrate biophysical changes along a finger length.

In 1876 Karl von Vierordt, who was a German physiologist, only observed changes in the solar spectrum transmitted by the finger tissues of his own hand. Today efficient selective transillumination of living tissues may be widely used in practice.
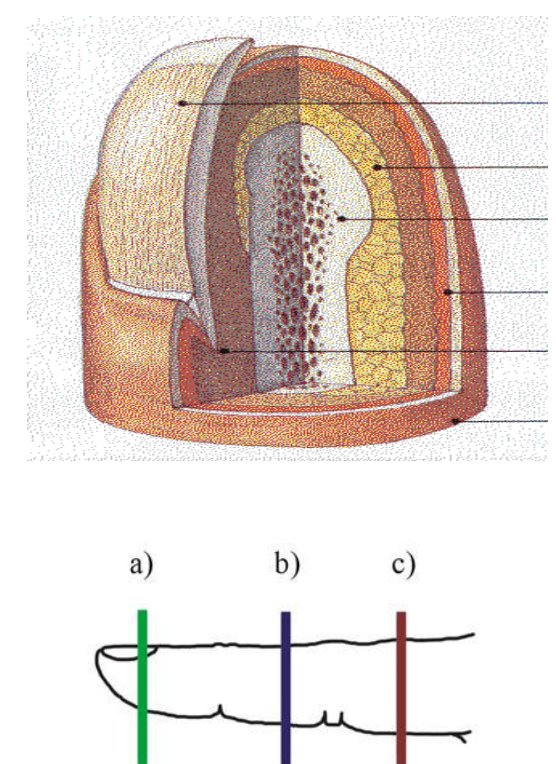

a)

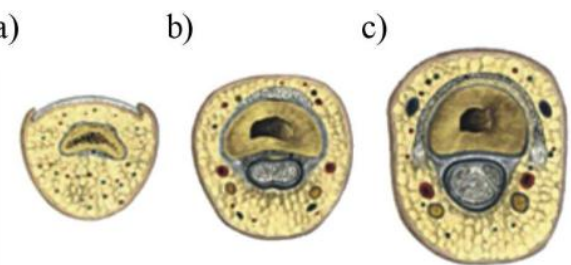

Fig. 2. Human fingertip structure to be transilluminated and human finger cross-sections [6].

Transillumination is understood as the phenomenon of transmitting optical radiation with defined parameters by an object, which becomes the carrier of information on the characteristic size of the object. The complicated object to be transilluminated may be virtually "reproduced" with a designed model based on the "sandwich" structure. This representation is performed with the division of a given object by virtual planes placed perpendicularly to the transillumination direction. The structure of a specialized algorithm which has been proposed makes possible creating a lot of free variants of reference standards, including changes in number of layers and their biophysical composition [6].
The simulation of different changes in finger tissue composition allows predicting the output results of occurring interactions. Experimental verification of the model structure was made with spectrophotometry.

The implemented model could be used in estimating the content and thickness of particular layers distinguished in a complex object and prediction of their transillumination efficiency. One of the important purposes of the presented simulation is evaluating the thickness of tissue at which discontinuity has to appear in intensity transmittance versus thickness. The point of transmittance discontinuity indicates a change in optical properties of a calculated layer, determining the intervals where a given transilluminated component of a tissue slab may be treated as optically thin or thick.

Pulse oximetry smartly combines the rules of both in vivo spectrophotometry with transmission or reflection photoplethysmography in monitoring arterial blood oxygen saturation. Continuous monitoring may be performed at rest, during activity or sleep. A novel expanded use of the transmission variant of a pulse oximetry concept proposed by the author depends on tracing two extreme components of raw optical signals, to be obtained from any blood-supplied tissues when illuminated with a given wavelength (Fig. 3) [7].
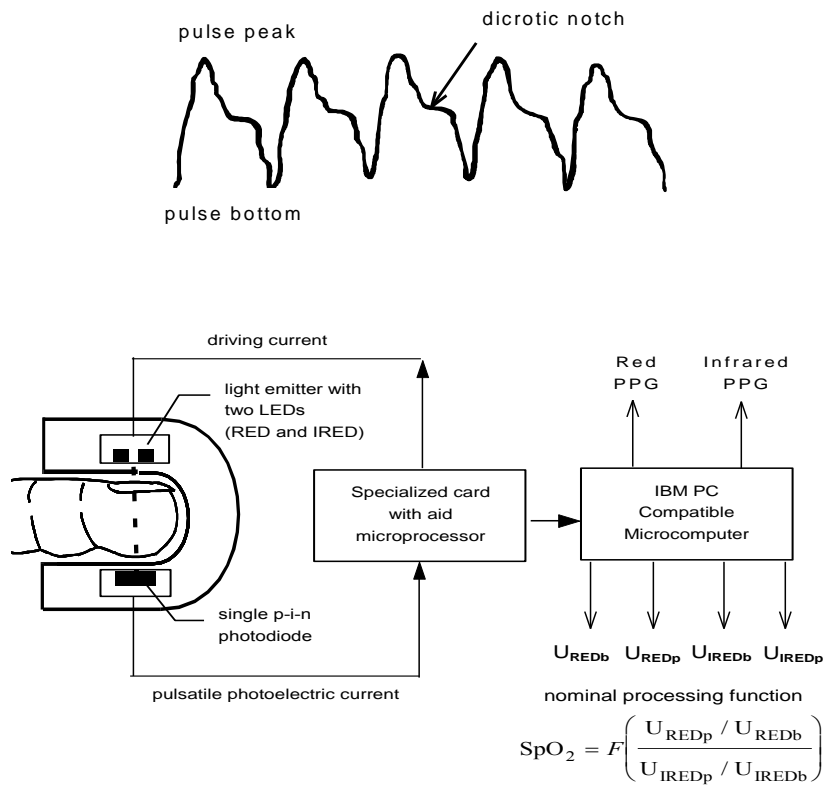

Fig. 3. Example of a blood pulse waveform and scheme illustrating measurements based on modified utilization of the transmission pulse oximetry concept to trace optical signals detected from an object [7].

The main component of the PPG curve is the arterial blood pulse waveform. Using the designed testing device, the components of two raw pulsatile optical signals from the finger tip, both obtained at wavelengths of 660 and $940 \mathrm{~nm}$ respectively, were converted to four values of 
voltage: $\mathrm{U}_{\mathrm{REDb}}$ and $\mathrm{U}_{\mathrm{IREDb}}$ representing a reference bottom of the arterial pulse wave in each heart beat, and $U_{\text {REDp }}$ and $\mathrm{U}_{\text {IREDp }}$ corresponding to the peak difference. The real relationship between oxygen saturation $\mathrm{SpO}_{2}$ and ratios: $\mathrm{U}_{\mathrm{REDp}} / \mathrm{U}_{\mathrm{REDb}}$ and $\mathrm{U}_{\text {IREDp }} / \mathrm{U}_{\text {IREDb }}$ has been determined experimentally. The author has made a broad set of experiments under laboratory as well as clinical conditions. A portion of studies was realized with the author as a patient, allowing her to obtain especially valuable data.

Monitoring of PPG curves connected with a cyclical change in blood volume pulsating in capillary vessels of the monitored body site can aid detection and control of disturbances in a circulatory rhythm. Transilluminated objects have to be optically thin. In the case of objects creating an optically thick layer, it is necessary to use reflectance sensors. A new multi-sensor approach to detecting peripheral reflectance PPG waveforms has been designed [8]. This system significantly expands the possibilities of analysis based on measuring data from several sensors placed in various points of human body. Particular sensor outputs can show the local shapes of a waveform of human heart beats. Some variants of sensors were designed and constructed (Fig. 4).
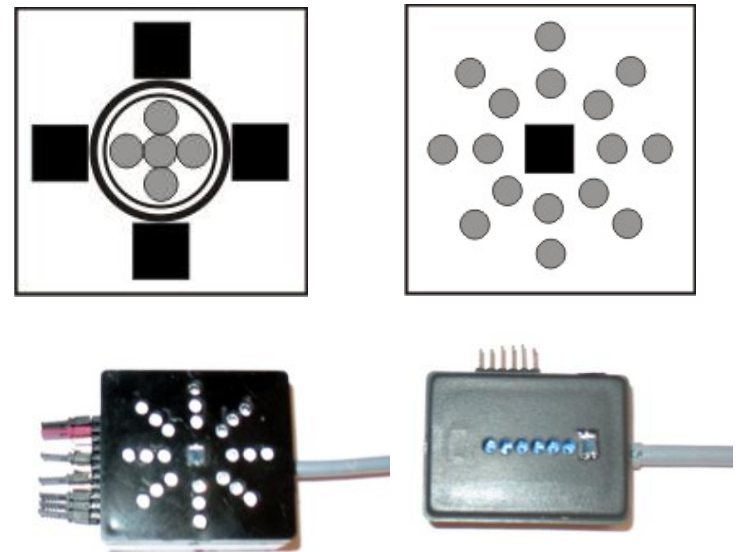

Fig. 4. Examples of the designed models of sensors; various versions of arrangement of light emitters and detectors have been tested [8].

Each sensor is equipped with a highly efficient light emitter (containing either a single LED or a set of LEDs) and a sensitive output light detector (containing either a single photodiode or a set of photodiodes). The system may cooperate with both reflectance and transmission sensors, so it is possible to distribute numerous sensors in various points of human body, each of different blood supply. A sensor may be attached to a given site for monitoring the beat-to-beat pulsation as well as shape of a peripheral pulse waveform. The shape of the PPG curve largely depends on the blood supply extent of the area examined. The numerical procedures enable a thorough analysis of the pulse wave concerning: the amplitude, time, and time as well as amplitude correlations between several signals. Data collected in this way may be useful in finding the reasons of cardiovascular action irregularities.

The interest in the development of optical techniques in biomedicine in order to obtain images of tissues and organs is great and still growing. Different imaging methods allow the detection of different properties of tissues through a variety of utilized phenomena. Thus, methods of medical imaging should not be treated as alternative but complementary to each other, according to the present tendencies in applying the so-called combined imaging [9-10]. Examples of optical monitoring with videoendoscopy during minimally invasive operations are shown in Figs. 5 and 6. Three successive images of gallstone removing are shown in Fig. 5, while three successive videoendoscopic images in Fig. 6 illustrate biliary duct stenting.

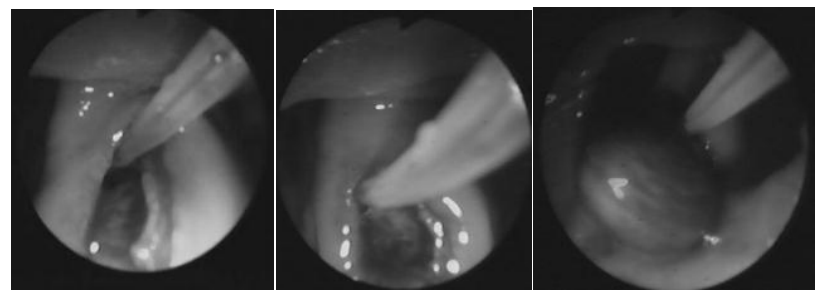

Fig. 5. Three successive images of gall-stone removing with videoendoscopy [10]
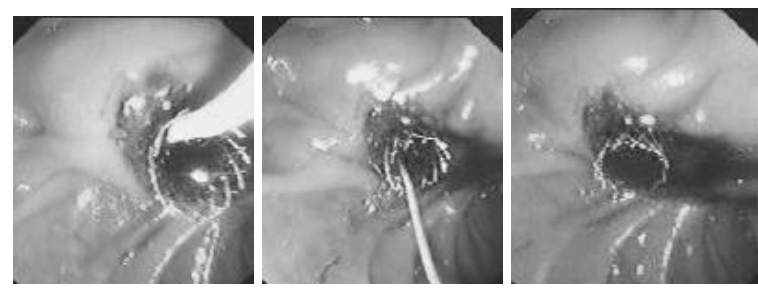

Fig. 6. Three successive videoendoscopic images of biliary duct stenting [9].

Optical techniques used in videoendoscopy supported by X-ray scanning plus percutaneous procedures allow access to pathological objects located in places inaccessible so far.

At present optical transillumination is used in monitoring blood oxygenation, hemorrhage detection, brain imaging, Alzheimer disease diagnostics, mammography and rheumatism and joint inflammable condition monitoring. Transillumination as the method of examination by the passage of light through tissues or a body cavity is a diagnostic technique in the course of intensive development at the moment.

Optical imaging has a lower resolution in comparison with, e.g., the RTG techniques, but allows to detect changes invisible on X-ray pictures (Fig. 7). 


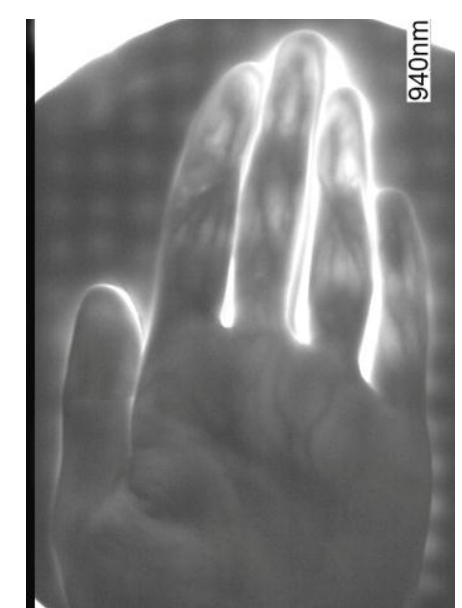

Fig. 7. Example of the fingers transillumination effect at radiation $940 \mathrm{~nm}$ emitted by a high power LED.

Biooptical signals are "difficult" both due to specific requirements related to the acquisition methods and the presence of numerous artifacts significantly impeding further digital conditioning. The current research was concentrated on mastering efficient transillumination of thick layers of tissues and building efficient and stable algorithms representing anatomic and functional properties.

The problems of image interpretation, with particular consideration of micro-images obtained from a CCD camera, were also undertaken [11]. Evaluation of parameters specifying the response of a virtual integrated oligonucleotide library was considered. The analysis has been concerned on fluorescence signals to be obtained as a result of hybridization and detected by a CCD camera equipped with an adjustable focal length optical system.

Other studies on stimuli design for the SSVEP-based brain computer-interface human brain reaction presented with stimuli flashing through a parallel analysis of SNR and comfort have determined that green is perceived as the most friendly colour by the users [12].

Furthermore, methods are developed [13], of identification of harmful electric power receivers with regard to dependence between variations of electrical signal supplying the light sources and oscillations of the light flux. The influence of voltage fluctuation on light flickering is important because humans can be sensitive to light flicker.

Experimental studies on a new approach to objective evaluation of human visual acuity have been also realized during human subjects examination under clinical conditions [14]. Visual acuity is a very important measure of spatial resolution of a visual processing system. Recently, interesting studies include psychophysical measurements of specific two-photon vision [15].
Of course, progressive development of noninvasive optical imaging and measurements always depends very much on clinical acceptance of a new advanced biomedical technology and engineering.

In conclusion of this short review, it may be stated that:

- The complementary use of optical imaging may provide information on the functional condition unavailable in other techniques of medical imaging;

- Optoelectronic photoplethysmography and pulse oximetry constitute the most significant technological advances made in clinical diagnostics since electrocardiography. These diagnostic techniques are still under intensive research. They concentrate on the development of effective transillumination of thick layers of tissues and on building efficient and stable algorithms representing and anatomic and functional properties. Numerous issues related to measurement result interpretation still remain unsolved. Thus, to enrich final readings with simultaneous tracing the extreme values of the processed biooptical signals are justifiable. A novel complementary look proposed by the author helps to state and evaluate the reasons for changes appearing in the transmitted selective optical signals;

- The long-terms plans of studies are connected with expected possibility to identify several changes in the features of biological objects exposed to selective illumination in the range of wavelength including visible and near infrared radiation.

\section{References}

[1] A. Cysewska-Sobusiak, Biocybernetics and Biomedical Engineering 22 (2002).

[2] J.G. Webster, Design of pulse oximeters (Bristol, IOP Publishing Ltd 1997).

[3] T. Aoyagi, Journal of Anesthesia 17, 4 (2003).

[4] A.A. Alian, K.H Shelley, Anesthesiology 28, 4 (2014).

[5] F.A. Duck, Physical properties of tissue: a comprehensive reference book (San Diego, Academia Press 1990).

[6] Z. Krawiecki, A. Cysewska-Sobusiak, G. Wiczyński, A. Odon, Bull. Pol. Ac. Tech. 56, 2 (2008).

[7] A. Cysewska-Sobusiak, Opt. Eng. 36, 4 (1997).

[8] D. Prokop, A. Cysewska-Sobusiak, and A. Hulewicz, Procedia Eng. 47 (2012).

[9] A. Cysewska-Sobusiak, P. Skrzywanek, and A. Sowier, IEEE Sensors Journal 6, 5 (2006).

[10] A. Cysewska-Sobusiak, G. Wiczyński, Z. Krawiecki, and A. Sowier, Opto-Electron. Rev. 16, 2 (2008).

[11] A. Cysewska-Sobusiak, M. Bołtrukiewicz, and J. Parzych, Optica Applicata 38, 2 (2008).

[12] M. Jukiewicz and A. Cysewska-Sobusiak, Intl. Journal of Electronics and Telecommunications 62, 2 (2016).

[13] G. Wiczyński, IEEE Trans. on Power Delivery, 27, 2 (2012).

[14] A. Hulewicz, Phot. Lett. Poland 6, 4 (2014).

[15] A. Zielińska, K. Kiluk, M. Wojtkowski, and K. Komar, Phot. Lett. Poland 11, 1 (2019). 